\title{
Editorial
}

\section{Radio Wave Propagation and Wireless Channel Modeling}

\author{
Ai Bo, ${ }^{1}$ Thomas Kürner, ${ }^{2}$ César Briso Rodríguez, ${ }^{3}$ and Hsiao-Chun $\mathrm{Wu}^{4}$ \\ ${ }^{1}$ State Key Laboratory of Rail Traffic Control and Safety, Beijing Jiaotong University, Beijing 100044, China \\ ${ }^{2}$ Technische Universität Braunschweig, Braunschweig 38106, Germany \\ ${ }^{3}$ Universidad Politecnica de Madrid, Madrid 28031, Spain \\ ${ }^{4}$ Louisiana State University, LA 70803, USA \\ Correspondence should be addressed to Ai Bo; aibo@ieee.org
}

Received 25 November 2012; Accepted 25 November 2012

Copyright (C) 2013 Ai Bo et al. This is an open access article distributed under the Creative Commons Attribution License, which permits unrestricted use, distribution, and reproduction in any medium, provided the original work is properly cited.

Mechanisms about radio wave propagation are the basis for wireless channel modeling. Typical wireless channel models for typical scenarios are of great importance to the physical layer techniques such as synchronization, channel estimation, and equalization. Nowadays, many channel models concerned with either large-scale modeling and small-scale fast fading models have been established. However, with the development of some new techniques such as multiuser MIMO systems, vehicle-to-vehicle technique, wireless relay technique, and short-distance or short-range technique, novel wireless channel models should be developed to cater for these new applications. As for this special issue, we cordially invite some researchers to contribute papers that will stimulate the continuing efforts to understand the mechanisms about radio wave propagations and wireless channel models under variant scenarios. This special issue provides the state-of-the-art research in mobile wireless channels.

As we know, scene partitioning is the premise and basis for wireless channel modeling. The paper titled "Radio wave propagation scene partitioning for high-speed rails" discusses the scene partitioning for high-speed rail (HSR) scenarios. Based on the measurements along HSR lines with $300 \mathrm{~km} / \mathrm{h}$ operation speed in China, the authors partitioned the HSR scene into twelve scenarios. Further work based on theoretical analysis of radio wave propagation mechanisms, such as reflection and diffraction, may lead to develop the standard of radio wave propagation scene partitioning for HSR.

The paper "Quantification of scenario distance within generic WINNER channel model" deals with the topic on the scenario comparisons on the basis of the fundamental theory that a generic WINNER model uses the same set of parameters for representing different scenarios. It approximates the
WINNER scenarios with multivariate normal distributions and then uses the mean Kullback-Leibler divergence to quantify the divergence. The results show that the WINNER scenario groups (A, B, C, D) or propagation classes (LoS, NLoS) do not necessarily ensure minimum separation within the groups/classes. The computation of the divergence of the actual measurements and WINNER scenarios confirms that the parameters of the C2 scenario in WINNER series are a proper reference for a large variety of urban macrocell environments.

The paper "Fading analysis for the high speed railway viaduct and terrain cutting scenarios" provides a good understanding of fading characteristics in HSR environment based on the measurements taken in both high-speed viaduct and terrain cutting scenarios using track side base stations. Kolmogorov-Smirnov (K-S) test has been first introduced in the statistical analysis to find out the most appropriate model for the small-scale fading envelope. Some important conclusions are drawn: though both Rice and Nakagami distributions provide a good fit to the first-order envelope data in both scenarios, only the Rice model generally fits the second-order statistics data accurately. For the viaduct scenario, higher Rice $\mathrm{K}$ factor can be observed. The change tendency of the $\mathrm{K}$ factor as a function of distance in the two scenarios is completely different.

The paper "Propagation mechanism modeling in the nearregion of arbitrary cross-sectional tunnels" talks about the modeling of the propagation mechanisms and their dividing point in the near-region of arbitrary cross-sectional tunnels. By conjunctively employing the propagation theory and the three-dimensional solid geometry, it presents a general model for the dividing point between two propagation mechanisms. 
Furthermore, the general dividing point model is specified in rectangular, circular, and arched tunnels, respectively. Five groups of measurements are used to justify the model in different tunnels at different frequencies. The results could help to deepen the insight into the propagation mechanisms in tunnels.

A key characteristic of train-to-train (T2T) communication, a recently proposed novel technique for HSR, is to avoid accidents conducted among trains without any aid of infrastructure. The paper "A novel train-to-train communication model design based on multihop in high-speed railway" provides a novel T2T communication model in a physical layer based on multihop and cooperation technique. The mechanism of this model lies in the idea that a source train uses trains on other tracks as relays to transmit signals to destination train on the same track. The paper titled "Outage analysis of train-to-train communication model over Nakagami-m channel in high-speed railway" analyzes the endto-end outage performance of T2T communication model in HSR over independent identical and nonidentical Nakagami$\mathrm{m}$ channels. It presents a novel closed form for the sum of squared independent Nakagami-m variates and then derives an expression for the outage probability of the identical and nonidentical Nakagami-m channels. Numerical analysis indicates that the derived analytical results are reasonable and the outage performance is better over Nakagami-m channel in HSR scenarios.

The above-mentioned papers are for HSR communication wireless channel models. There are also some papers dealing with the channel models for satellites, ships, human bodies, and vehicle-to-vehicle communications.

The paper "State modeling of the land mobile propagation channel with multiple satellites" evaluates a novel approach for multisatellite state modeling: the Master-Slave approach and its corresponding realization method named Conditional Assembling Method. The primary concept is that slave satellites are modeled according to an existing master state sequence, whereas the correlation between multiple slaves is omitted. For modeling two satellites (one master and one slave), the "Conditional Assembling Method" enables an accurate resimulation of the correlation coefficient between the satellites, the single satellite state probabilities, and the combined state probabilities of master and slave. The probability of the "all bad-" state resulting from masterslave is compared with an analytically estimated "all bad-" state probability from measurements. Master-slave has a high probability error in case of a high correlation between the slave satellites. Furthermore, a master satellite with a high elevation provides a lower probability error compared to a master with low elevation.

The improvement of maritime radio links often requires an increase of emitted power or receiver sensitivity. Another way is to replace the poor antenna gains of traditional surface ship whips by novel antenna structures with directive properties. The paper "Impact of ship motions on maritime radio links" developed a tool for modeling the impact of ship motions on the antenna structures. The tool includes a deterministic two-ray model for radio-wave propagation over the sea surface.
The paper "Statistical modeling of ultrawideband bodycentric wireless channels considering room volume" presents the results of a statistical modeling of on-body ultrawideband (UWB) radio channels for wireless body area network (WBAN) applications. A measured delay profile can be divided into two domains: in the first domain there is either a direct (for line of sight) or diffracted (for nonline of sight) wave which is dependent on the propagation distance along the perimeter of the body, but essentially unrelated to room volume; and the second domain has multipath components that are dominant and dependent on room volume. The first domain was modeled with a conventional power decay law model and the second domain with a modified SalehValenzuela model considering the room volume. Realizations of the impulse responses are presented based on the composite model and compared with the measured average power delay profiles.

The paper titled "On the statistical properties of Nakagamihoyt vehicle-to-vehicle fading channel under nonisotropic scattering" argues the statistical properties of vehicle-to-vehicle Nakagami-Hoyt (Nakagami-q) channel model under nonisotropic condition. The spatial-time correlation function (STCF), the power spectral density (PSD), squared time autocorrelation function (SQCF), level crossing rate (LCR), and the average duration of fade (ADF) of the NakagamiHoyt channel have been derived under the assumption that both the transmitter and receiver are nonstationary with nonomnidirectional antennas. A simulator utilizing the inverse-fast-fourier-transform- (IFFT-) based computation method is designed for the model.

The paper "NECOP propagation experiment: rain-rate distributions observations and prediction model comparisons" talks about the empirical distribution functions for the evaluation of rain-rate based on the observed data. The empirical distribution functions were compared with cumulative distribution functions generated using four different rain-rate distribution models. It is found that although each of the models shows similar qualitative features at lower exceedance of time, the characteristics at higher time percentages show quantitative difference from the experimental data except the improved version of Moupfouma model. The results further show that the rain-fall rate and the microwave propagation characteristics in the observed region are out of accord with International Telecommunication Union predictions. This information is vital for predicting rain fading cumulative probability distributions.

The paper "Influence of training set selection in artificial neural network-based propagation path loss predictions" utilizes the artificial neural networks (ANNs) and ray-tracing technique for predicting the received power/path loss in both outdoor and indoor links. A complete description of the process for creating and training an ANN-based model is presented with special emphasis on the training process. The optimum selection of the training set is discussed. A quantitative analysis based on results from two narrowband measurement campaigns is also presented.

The following fours papers are related with multi-input and multioutput (MIMO) techniques. The paper " $A$ tradeoff 
between rich multipath and high receive power in MIMO capacity" presents a discussion of rich multipath (in NLOS) or signal-to-noise ratio (SNR) (usually in LOS) effects on MIMO channel capacity. It is investigated by performing simulations using simple circle scatterer and WINNER II channel model. The simulation results show that these two factors behave differently as the channel conditions vary. When the scatterer number in channel is low, the high receive SNR is more important than capacity. The multipath richness will get the upper bound when the scatterer number is beyond a certain threshold. However, the channel capacity will not change much as the scatterers continue to increase.

The paper "Construction and capacity analysis of highrank LoS MIMO channels in high speed railway scenarios" talks about the validity of the maximum capacity criterion applied to realize high-rank LoS MIMO channels for HSR scenarios. Performance is evaluated by ergodic capacity. Numerical results demonstrate that, by simply adjusting antenna spacing according to the maximum capacity criterion, significant capacity gains are achievable. Two proposals are presented to reconfigure antenna arrays so as to maximize LoS MIMO capacity in the HSR scenarios. The paper "Geometry-based stochastic modeling for MIMO channel in high-speed mobile scenario" discusses the geometry-based stochastic channel models for the terrain cutting, suburb, and urban scenarios in HSR. The space time correlation functions in analytical form are obtained in suburb and urban scenarios. The comparisons of the space correlation characteristics under three scenarios are made.

The paper "Performance evaluation of closed-loop spatial multiplexing codebook based on indoor MIMO channel measurement" discusses closed-loop MIMO technique standardized for long-term evolution (LTE) system. Based on the wideband MIMO channel measurement in a typical indoor scenario, capacity loss (CL) of the limited size codebook relative to perfect precoding is studied in two extreme channel conditions. The results show that current codebook design for single-layer transmission is nearly capacity lossless and the CL will increase with the number of transmitted layers. Furthermore, the capacity improvement of better codebook selection criterions is very limited compared to CL. To survey the effect of frequency domain channel variation on MIMO-OFDM system, a function is defined to measure the fluctuation levels of the key channel metrics within a subband and to reveal the inherent relationship between them.

Ai Bo

Thomas Kürner

César Briso Rodríguez Hsiao-Chun Wu 

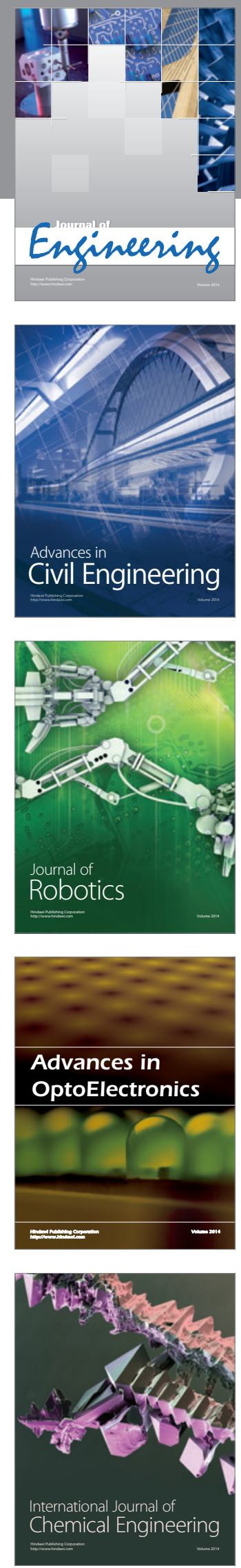

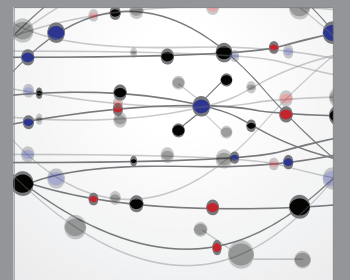

The Scientific World Journal
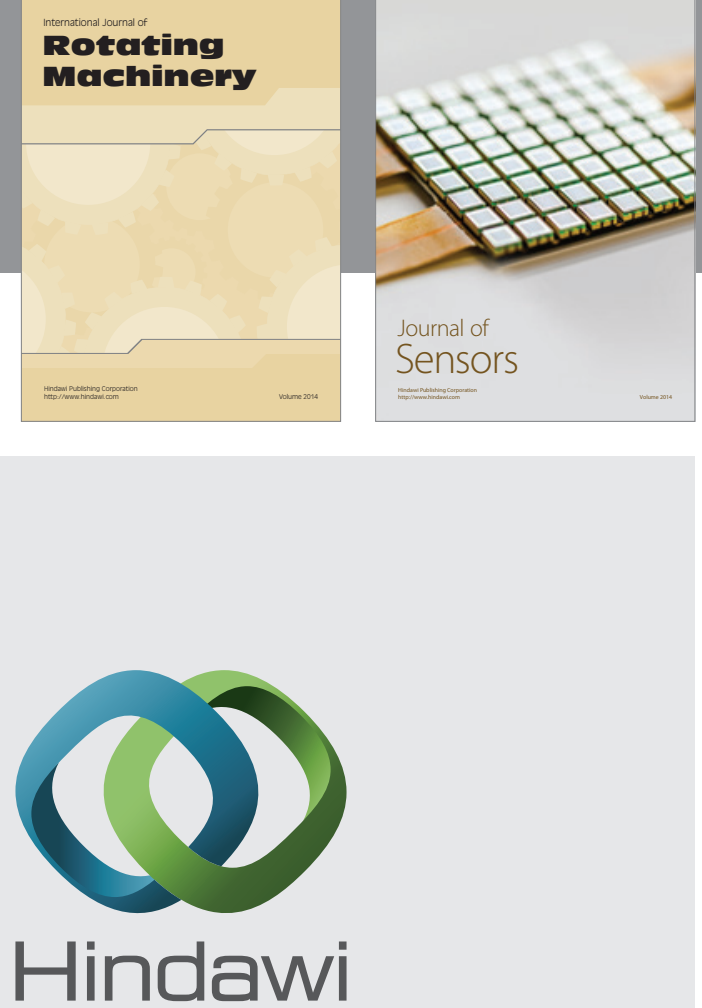

Submit your manuscripts at http://www.hindawi.com
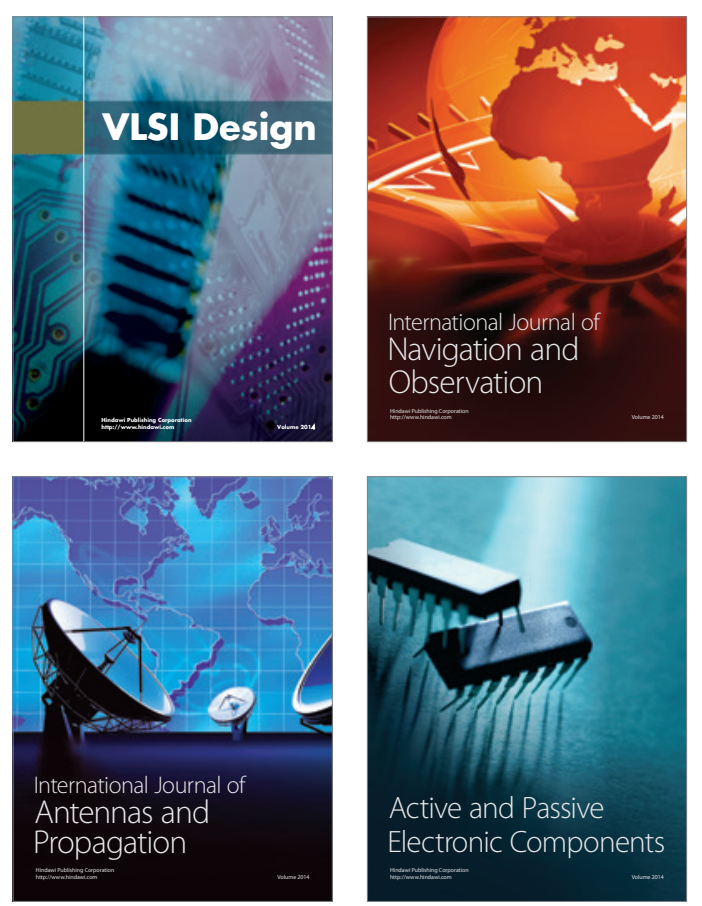
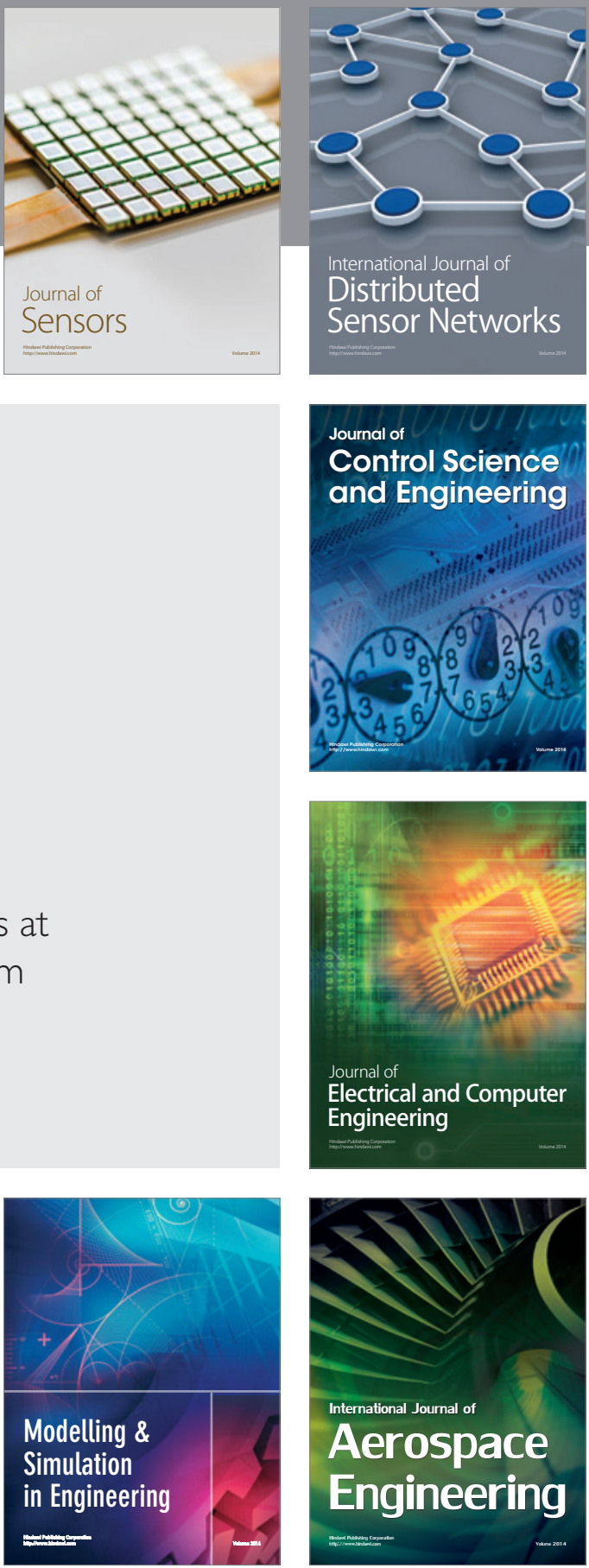

Journal of

Control Science

and Engineering
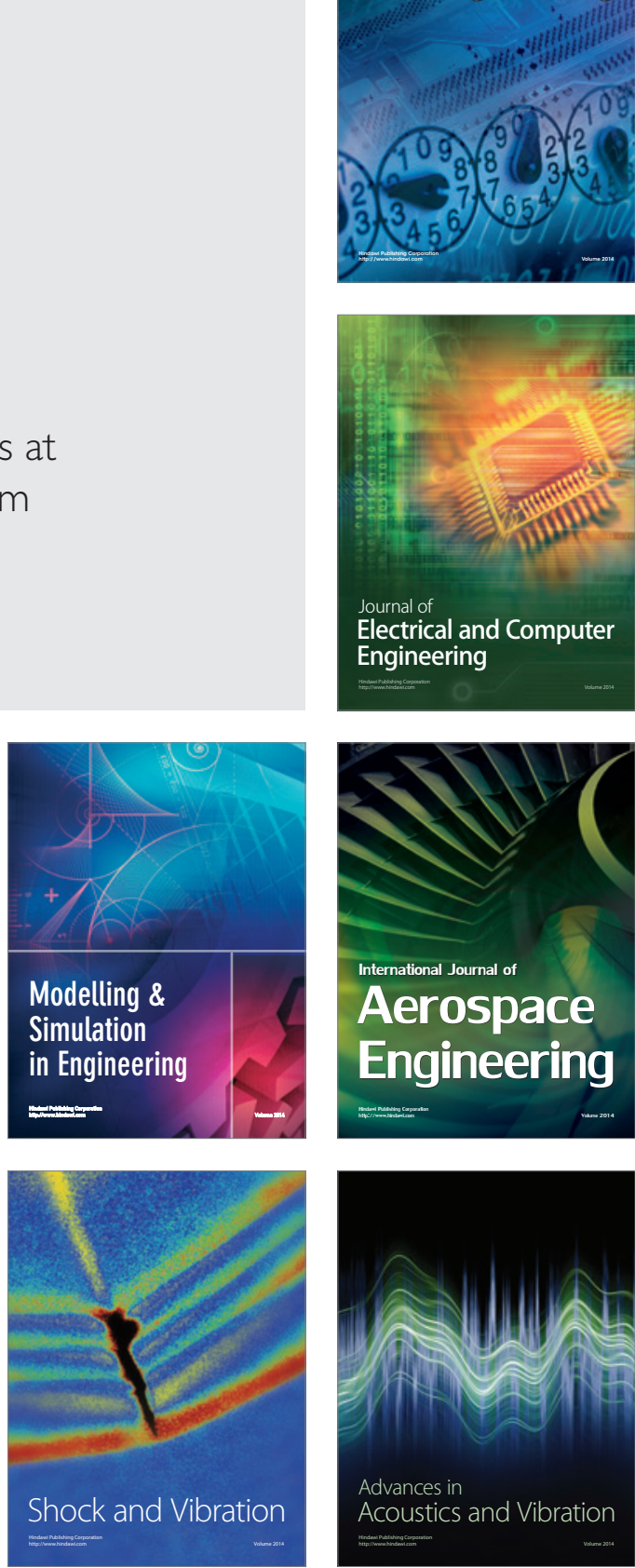